Discussion Paper No. 06-007

Combining Top-Down and Bottom-up in Energy Policy Analysis:

A Decomposition Approach

Christoph Böhringer and Thomas F. Rutherford

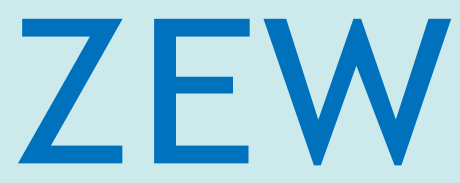

Zentrum für Europäische Wirtschaftsforschung $\mathrm{GmbH}$

Centre for European

Economic Research 
Discussion Paper No. 06-007

\section{Combining Top-Down and Bottom-up in Energy Policy Analysis: A Decomposition Approach}

Christoph Böhringer and Thomas F. Rutherford

Download this ZEW Discussion Paper from our ftp server:

ftp://ftp.zew.de/pub/zew-docs/dp/dp06007.pdf

Die Discussion Papers dienen einer möglichst schnellen Verbreitung von neueren Forschungsarbeiten des ZEW. Die Beiträge liegen in alleiniger Verantwortung der Autoren und stellen nicht notwendigerweise die Meinung des ZEW dar.

Discussion Papers are intended to make results of ZEW research promptly available to other economists in order to encourage discussion and suggestions for revisions. The authors are solely responsible for the contents which do not necessarily represent the opinion of the ZEW. 


\section{Nontechnical Summary}

The combination of bottom-up and top-down approaches constitutes a long-standing challenge in applied energy policy analysis. The terms "top-down" and "bottom-up" are shorthand for aggregate and disaggregated models. Top-down models examine the broader economy and incorporate feedback effects between different markets triggered by policy-induced changes in relative prices and incomes. They typically do not feature technological details of energy production or conversion. Energy sectors - like other non-energy sectors - are mostly represented in an aggregate way by means of smooth production functions which capture substitution (transformation) possibilities via substitution (transformation) elasticities. As a consequence, conventional top-down models cannot readily incorporate different assumptions about how discrete energy technologies and costs will evolve in the future; top-down models may also violate fundamental physical restrictions such as the conservation of matter and energy. In contrast, bottom-up models -- usually cast as mathematical programming problems - describe current and prospective technologies in detail. They are therefore well suited to the analysis of specific changes in technology or command-and-control policies such as efficiency standards. A common shortcoming of the bottom-up analysis is that it fails to account for price distortions, economy-wide interactions and income effects.

The formulation of economic equilibrium conditions as mixed complementarity problem (MCP) provides a unifying framework for combining technological details of bottom-up models and economic richness of top-down models. However, dimensionality may impose significant limitations on the practical application of the integrated MCP framework, particularly when the underlying programming formulation of the energy system includes both upper and lower bounds on many decision variables.

In this paper, we present a decomposition approach that combines different mathematical formats mixed complementarity and mathematical programming - thereby taking advantage of efficient and robust solution algorithms for each format: We use complementarity methods to solve the top-down economic equilibrium model and quadratic programming to solve the underlying bottom-up energy supply model. Our decomposition technique overcomes dimensionality restrictions of the integrated MCP framework and can be readily applied to the combination of large-scale top-down and bottom-up models for comprehensive energy policy analysis. 


\title{
Combining Top-Down and Bottom-Up in Energy Policy Analysis:
}

\section{A Decomposition Approach}

\author{
Christoph Böhringer \\ Centre for European Economic Research (ZEW), Mannheim, Germany \\ Department of Economics, University of Heidelberg, Germany \\ and \\ Thomas F. Rutherford \\ Ann Arbor, MI, U.S.A.
}

\begin{abstract}
The formulation of market equilibrium problems as mixed complementarity problems (MCP) permits integration of bottom-up programming models of the energy system into top-down general equilibrium models of the overall economy. Despite the coherence and logical appeal of the integrated MCP approach, implementation cost and dimensionality both impose limitations on its practical application. A complementarity representation involves both primal and dual relationships, often doubling the number of equations and the scope for error. When an underlying optimization model of the energy system includes upper and lower bounds on many decision variables the MCP formulation may suffer in robustness and efficiency. While bounds can be included in the MCP framework, the treatment of associated income effects is awkward.

We present a decomposition of the integrated MCP formulation that permits a convenient combination of top-down general equilibrium models and bottom-up energy system models for energy policy analysis. We advocate the use of complementarity methods to solve the top-down economic equilibrium model and quadratic programming to solve the underlying bottom-up energy supply model. A simple iterative procedure reconciles the equilibrium prices and quantities between both models. We illustrate this approach using a simple stylized model.
\end{abstract}

JEL classification: C61, C68, D58, Q43

Keywords: Mathematical Programming, Mixed Complementarity, Top-Down/Bottom-Up 


\section{Introduction}

Top-down and bottom-up are the two modeling paradigms to represent interactions between the energy system and the economy (International Panel on Climate Change (IPCC) [1996]). The terms "top-down" and "bottom-up" are shorthand for aggregate and disaggregated models. Models in the first category emphasize economy-wide, while those in the second category feature sectoral and technological details. The dichotomy of energy-economy models into top-down and bottom-up approaches is sometimes traced back to competing paradigms. It should be noted, however, that differences are less of conceptual nature, i.e. due to controversial theoretical underpinnings, but simply relate to the different levels of sectoral and technological aggregation as well as to the scope of ceteris paribus assumptions.

Top-down models examine the broader economy and incorporate feedback effects between different markets triggered by policy-induced changes in relative prices and incomes. They typically do not feature technological details of energy production or conversion. Energy sectors - like other non-energy sectors - are mostly represented in an aggregate way by means of smooth production functions which capture substitution (transformation) possibilities via substitution (transformation) elasticities. As a consequence, conventional top-down models cannot readily incorporate different assumptions about how discrete energy technologies and costs will evolve in the future; top-down models may also violate fundamental physical restrictions such as the conservation of matter and energy.

In contrast, bottom-up models - usually cast as mathematical programming problems describe current and prospective technologies in detail. They are therefore well suited to the analysis of specific changes in technology or command-and-control policies such as efficiency standards. A common shortcoming of the bottom-up analysis is that it fails to account for price distortions, economy-wide interactions and income effects (e.g. revenue recyling effects of energy taxes).

There are various hybrid modeling efforts that aim at combining the technological explicitness of bottom-up models with the economic richness of top-down models. These efforts can be broadly classified into two approaches. The first approach attempts to couple existing large-scale bottom-up and top-down models (e.g. Hudson and Jorgenson [1974], Bergmann [1990]). Due to the heterogeneity in complexity and accounting methods across the sub-models such a "soft-link" approach may face substantial problems in achieving overall consistency and convergence of iterative solution algorithms. ${ }^{1}$ The second approach puts strong emphasis on overall economic consistency and therefore makes use of a single integrated modeling framework in order to "hard-link" bottom-up and top-down features. Such an integrated framework is provided by the specification of market equilibrium models as mixed complementarity problems (MCP - see Cottle and Pang [1992], Rutherford [1995]).

\footnotetext{
${ }^{1}$ Manne and Rutherford [1994] employ iterative methods to incorporate general equilibrium effects within the context of technology-oriented bottom-up models.
} 
The explicit representation of weak inequalities and complementarity between decision variables and market equilibrium conditions in the MCP formulation permits the modeler to capture both technological details and economic richness in a single mathematical format (Böhringer [1998]). More recently, the availability of robust large-scale solvers for MCP problems (Dirkse and Ferris [1995]) has promoted the implementation and application of hybrid energy-economy models in the MCP format to concrete energy regulation policies (e.g. Böhringer et al. [2003] or Frei et al. [2003]).

Despite the coherence and logical appeal of the integrated MCP approach, algebraic complexity and dimensionality impose significant limitations on its practical application, when the optimization problem describing the energy system includes upper and lower bounds on many decision variables. Bounds may also be incorporated in the MCP framework but the representation of associated income effects becomes rather problematic.

In this paper, we propose a decomposition technique to overcome such dimensionality restrictions. We describe how an integrated MCP model can be decomposed and solved iteratively: Complementarity methods are used to solve the top-down economic equilibrium model and quadratic programming is applied to solve the underlying bottom-up energy (supply) model. We present a worked example which illustrates the usefulness of the energysector decomposition, both in terms of efficiency of computation and transparency of model formulation. Convergence of our iterative (Jacobi) algorithm requires that the decomposed energy sector be small relative to the rest of the economy. In this setting, a Marshallian demand approximation in the energy sector model provides a precise local representation of the general equilibrium demand so that overall convergence occurs rapidly. ${ }^{2}$

The remainder of this paper is organized as follows. In section 2, we extend the top-down representation of economic market equilibria as a mixed complementarity problem by an explicit bottom-up linear programming sub-model of energy supply. In section 3, we lay out how the integrated top-down / bottom-up MCP model can be decomposed. In section 4, we provide an illustrative numerical example. In secton 5 , we conclude.

\section{Integrated Model Formulation}

Market equilibrium features complementarity between upper and lower bounds on equilibrium variables and the weak inequalities which characterize market equilibrium. The complementarity features of market equilibria motivate the mathematical formulation of market equilibrium problems as a mixed complementarity problem (MCP). The MCP approach provides a general mathematical format that covers weak inequalities, i.e. a mixture

\footnotetext{
${ }^{2}$ In applications in which the energy system turns out to be of sufficient significance that the decomposition is costly or unreliable, our use of a complementarity formulation for the general equilibrium model permits selected features of the energy sector to be computed simultaneously with the macro-economy; the remaining part of the energy systen can then still be solved as a quadratic programming problem.
} 
of equations and inequalities, and complementarity between variables and functional relationships. It includes a wide range of mathematical problems including systems of linear or nonlinear equations or mathematical programs (Rutherford [1995]). The MCP formulation relaxes the integrability constraints for equilibrium conditions which emerge as first-order conditions from primal or dual optimization problems. This permits the direct representation of market inefficiencies such as distortionary taxes or spillovers that cannot be readily studied in an optimization framework (Böhringer and Rutherford [2005]).

In our formulation of an integrated top-down / bottom-up model we consider a competitive (Arrow-Debreu) economy with $n$ commodities (including economic goods, energy goods and primary factors) indexed by $i, m$ production activities (sectors) indexed by $j$, and $h$ households (including government) indexed by $k$. We extend the MCP framework suggested by Mathiesen [1985] by embedding an explicit linear-programming sub-model of energy supply in the economy. The decision variables of the economy can be classified into the following categories:

$p$ denotes a non-negative $n$-vector in prices for all goods and factors,

$y$ is a non-negative $m$-vector for activity levels of constant-returns-to-scale (CRTS) production sectors,

$M$ is a $h$-vector of consumer income levels,

$e$ represents a non-negative $n$-vector of net energy system outputs (including, for example, electricity, oil, coal, and natural gas supplies to residential, industrial, and commercial customers), and

$x$ denotes a non-negative $n$-vector of energy system inputs (including labor, capital, and materials inputs).

As in Mathiesen's model a competitive market equilibrium for this economy is represented by a vector of activity levels, a non-negative vector of prices, and a non-negative vector of incomes such that:

- No production activity makes a positive profit (zero-profit condition): ${ }^{3}$

$$
-\Pi_{j}(p) \geq 0
$$

in which $\Pi_{j}(p)$ denotes the unit profit function for CRTS production activity $j$, which is calculated as the difference between unit revenue and unit cost (i.e., $\Pi_{j}(p)=r_{j}(p)-$ $\left.c_{j}(p)\right)$.

\footnotetext{
${ }^{3}$ We omit tax distortions from this exposition solely to economize on notation. An important and appealing feature of the top-down general equilibrium framework is its ability to address the welfare consequences of energy policy from a public finance perspective, i.e. accounting for initial tax distortions and public sector budget constraints.
} 
- Excess supply (supply minus demand) is non-negative for all goods and factors (market clearance condition):

$$
\sum_{j} \nabla \Pi_{j}(p) y_{j}+\sum_{k} \omega_{k}+e \geq \sum_{k} d_{k}\left(p, M_{k}\right)+x
$$

in which $\omega_{k}$ indicates the initial endowment vector for household $k$ and $d\left(k, M_{k}\right)$ is the utility-maximizing demand vector for household $k$.

- Expenditure does not exceed income (budget constraint):

$$
M_{k}=p^{T}\left[\omega_{k}+\theta_{k}(e-x)\right]
$$

in which $\theta_{k}$ represents the share of energy-sector rents that accrue to household $k$ (rents depend on household ownership of energy resources). The equations which define consumer income levels are conceptually distinct from the zero profit and market clearing conditions in that there is no explicit complementarity at work in this part of the model. The income variables are added to the equilibrium system solely as a means of simplifying the expression of household demand.

Furthermore, we assume that the equilibrium levels of energy sector outputs and inputs are consistent with profit-maximization, taking market prices as given:

- Energy sector supply and demand vectors are profit-maximizing choices subject to technical constraints. That is, $e$ and $x$ solve a linear programming model. For concreteness, we will assume that this bottom-up model can be written as:

$$
\max p^{T}(e-x)
$$

subject to

$$
\begin{gathered}
A x+B z \geq C e \\
e, x \geq 0, \quad \ell \leq z \leq u
\end{gathered}
$$

in which $A, C \in R^{M \times n}$, and $B \in R^{M \times N}$ characterize technical constraints and $z \in R^{N}$ denotes decision variables of the energy system.

In the integrated model formulation the linear program can be incorporated through the associated Kuhn-Tucker conditions and solved simultaneously with the equilibrium conditions (1)-(3):

$$
\begin{gathered}
C^{T} \pi \geq p, \quad e \geq 0, \quad e^{T}\left(C^{T} \pi-p\right)=0 \\
p \geq A^{T} \pi, \quad x \geq 0, \quad x^{T}\left(p-A^{T} \pi\right)=0 \\
A x+B z \geq C e, \quad \pi \geq 0, \quad \pi^{T}(A x+B z-C e)=0 \\
\ell \leq z \leq u, \quad \lambda, \mu \geq 0, \quad \lambda(z-\ell)=0, \quad \mu(u-z)=0 \\
\lambda+B^{T} \pi=\mu
\end{gathered}
$$


The attribution of energy-sector rents to households can be made explicit by writing (3) as:

$$
M_{k}=p^{T} \omega_{k}+\Theta_{k}\left(\mu^{T} u+\lambda^{T} \ell\right)
$$

in which $\Theta_{k} \in R^{h \times N}$ determines rents on energy-sector resources to households.

The integrated equilibrium for this hybrid top-down / bottom-up model then consists of $m+3 n+h+M+3 N$ equations as compared with the standard economic model of dimension $m+n+h$ and the original linear programming model with $M$ constraints and $N+2 n$ variables. $^{4}$ If computational complexity increases superlinearly with the problem dimension, a decomposition approach can outperform a simultaneous solution algorithm, provided that sufficiently few outer iterations are required to solve the decomposed model.

\section{A Decomposition Strategy}

The introduction of an energy-sector sub-model within the general equilibrium framework poses computational challenges when the dimensionality of the energy sector - captured by $N+M$ in our abstract setting - is very large relative to the dimensionality of the standard economic model given by $n+m+h$. While an integrated MCP formulation is attractive for highly aggregated energy system representations, it has limited practicality for large-scale systems with bounds on many variables due to the need to account for associated income effects. The added dimensionality poses two problems. First, the enlarged system may be more difficult to solve. Second, and more importantly in our view, the model is more awkward to implement because of the larger number of variables and equations as well as the complexity of explicit income constraints. Compactness of representation leads to fewer potential sources of error and more rapid model development. Calendar time rather than CPU time is the primary concern of the applied analyst.

In view of the challenges presented by the complementarity format we propose a decomposition strategy for the integrated model in which the energy system bottom-up component can be computed separately from the top-down economic general equilibrium sub-model. The solution procedure involves iterative solution of the top-down general equilibrium model given net supplies from the bottom-up energy sector sub-model followed by the solution of the energy sector sub-model based on a locally calibrated set of demand functions for energy sector outputs. When $(e-x)$ and $\theta$ are given exogenously, the top-down general equilibrium model can be solved as a complementarity problem of dimension $m+n+h$.

Suppose that the computed equilibrium prices are $\bar{p}$ (based on an initial guess for the energy sector response $\bar{e}, \bar{x}$, and $\bar{\theta})$. The next step in a recursive solution procedure updates the values of $(e-x)$ and $\theta$ based on $\bar{p}$. One might then consider a direct solution of the

\footnotetext{
${ }^{4}$ The linear programming model represents upper and lower bounds on decision variables through implicit Kuhn-Tucker conditions, such that we can reduce the number of constraints by $2 N$ as compared with the integrated MCP formulation.
} 
profit-maximizing linear program (4) which characterizes the choices of an individual firm. However, this approach is quite likely to fail because the profit-maximizing linear program does not take account of the response of market demand to changes in energy prices. Suppose that these demand elasticities are given by $\epsilon{ }^{5}$ We can then write the demand for energy good $i$ as:

$$
e_{i}(p)=\bar{e}_{i}\left[1-\epsilon_{i}\left(p_{i} / \bar{p}_{i}-1\right)\right]
$$

where $\epsilon_{i}$ is the demand elasticity and $\bar{e}_{i}$ and $\bar{p}_{i}$ denote the observable reference quantities and prices for the demand function calibration.

The calibrated inverse demand function is:

$$
p_{i}(e)=\bar{p}_{i}\left[1-\left(1-e_{i} / \bar{e}_{i}\right) / \epsilon_{i}\right]
$$

and the integrated market demand function is:

$$
\int p_{i}\left(e_{i}\right) d e_{i}=\bar{p}_{i} e_{i}\left[1-\frac{e_{i}-2 \bar{e}_{i}}{2 \epsilon_{i} \bar{e}_{i}}\right],
$$

Am aggregate (integrated) multicommodity energy systemn may then be solved as a quadratic programming problem of the form: ${ }^{6}$

$$
\max \bar{p}^{T}(e-x)-\frac{1}{2} \sum_{i} \frac{\bar{p}_{i} e_{i}}{\epsilon_{i} \bar{e}_{i}}\left(e_{i}-2 \bar{e}_{i}\right)
$$

subject to the same constraints which appear in (4) in order to compute a partial market equilibrium model for the energy sector based on linear demand functions locally calibrated to the given macroeconomic equilibrium.

Figure 1 illustrates the basic steps involved in the iterative model solution. The top-down model is solved as a complementarity problem, taking net energy supplies $\left(\bar{e}_{i}\right)$ and energy sector inputs $(\bar{x})$ as given. The computed equilibrium determines prices $\left(\bar{p}_{i}\right)$ and a set of linear demand curves for energy sector outputs $\left(D_{i}(p ; \epsilon)\right)$. These demand curves and relative prices parameterize the bottom-up model which may be solved as a quadratic program.

We graphically portray the iterative solution process in Figures 2 and 3. Figure 2 sketches a simplified partial equilibrium model where we neglect general equilibrium and cross-market price effects. Here, we essentially use the decomposition procedure to compute a singlesector Marshallian market equilibrium with a nonlinear demand curve $(D D)$ and a piecewise linear supply schedule $(S)$. The starting point of the algorithm is an initial estimate $Q_{0}$ of the quantity of energy supply. This quantity has an associated market price (marginal

\footnotetext{
${ }^{5}$ If - as is commonly the case - the general equilibrium model is based on nested constant-elasticity-ofsubstitution technologies and preferences, the demand elasticity of individual market segments is roughly equal to the elasticity of substitution.

${ }^{6}$ We assume in this algorithm that the energy sector faces constant prices for non-energy economic inputs, which is a reasonable assumption considering that the energy sector typically comprises at most $5 \%$ of the overall economic activity.
} 


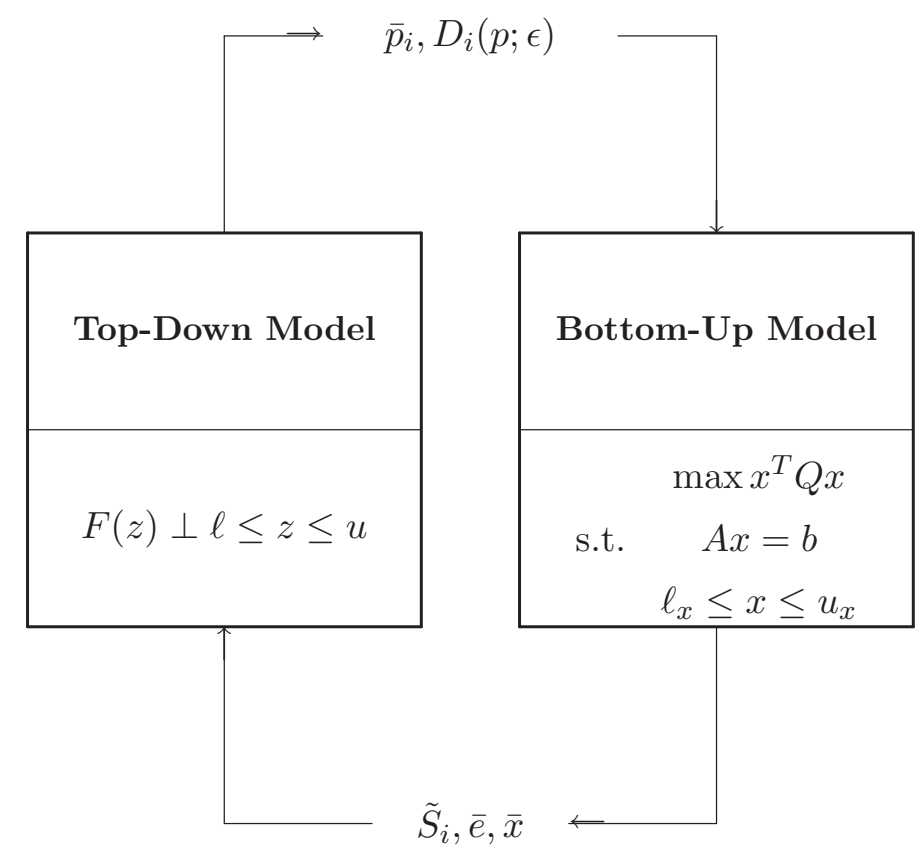

Figure 1: Iterative Decomposition Algorithm

willingness to pay) of $P_{0}$ and market equilibrium point $a$. Having computed this equilibrium (ignoring the supply schedule), the algorithm next evaluates the energy market based on a linear demand curve calibrated to the market equilibrium at point $a$. The solution to the supply problem maximizes the sum of consumer and producer surplus (the shaded area), resulting in an equilibrium supply of $Q_{1}$ at a marginal cost of $c_{1}$. Given $Q_{1}$, the algorithmic steps are repeated to converge at the equilibrium solution $\left(P^{*}, Q^{*}\right)$.

Figure 3 illustrates the same procedure for a multi-market general equilibrium in which shifts in the demand for one good induce changes in the demand for other goods through general equilibrium income and cross-market price effects. At the benchmark point we have an energy demand function $D_{0}-D_{0}$ which is locally approximated by a linear demand function at point $a$. At the next iteration, changes in energy supplies and associated general equilibrium effects lead to the energy demand function $D_{1}-D_{1}$ which is approximated locally at $b$. 


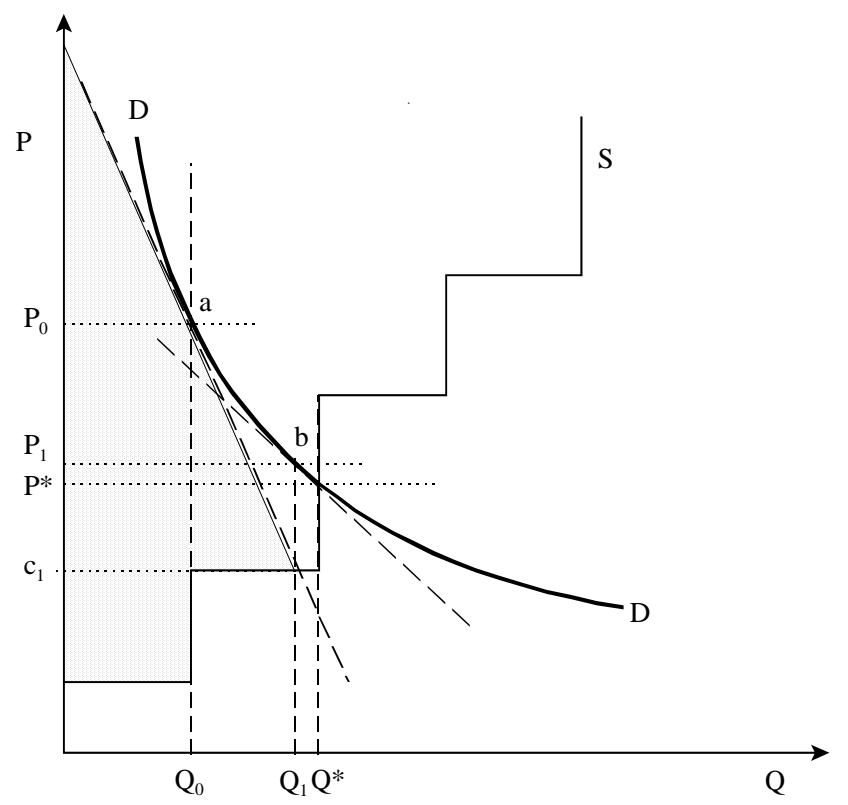

Figure 2: Iterative Adjustments: Single Market Partial Equilibrium

\section{An Illustrative Example}

As illustration of the algorithm's implementation and computational efficiency, we have formulated a stylized representative agent model with two non-energy goods $(x, y)$ and a set of four energy goods (OIL, GAS, COL (coal), and ELE (electricity)). We begin with an algebraic characterization of our model in a primal optimization setting. We then provide a re-formulation of the model as a mixed complementarity problem. Next, we lay out the decomposition of the integrated top-down/bottom-up model. Finally, we lay out the decomposition of the integrated top-down/bottom-up model, describing how such a model can be calibrated to base-year social accounts.

\subsection{The Integrated Model in a Primal Optimization Setting}

Energy goods are produced by a discrete number of technologies. ${ }^{7}$ Aggregate supply $E_{i}$ of energy good $i$ equals output $z_{i t}$ from all technologies $t$ producing that energy good $i$ :

$$
E_{i}=\sum_{t} z_{i t}
$$

\footnotetext{
${ }^{7}$ In our numerical model implementation, we can vary the number of technologies available for producing each energy good in order to perform sensitivity analysis of computational efficiency with respect to the dimensionality of the energy system sub-model (see Figure 5).
} 


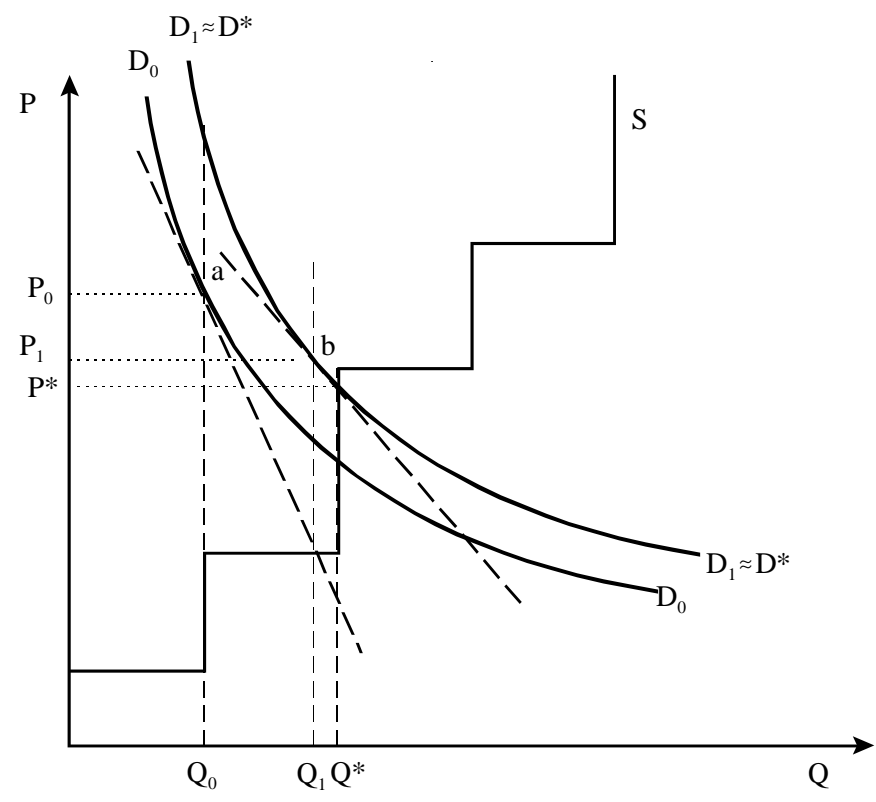

Figure 3: Iterative Adjustments: Multimarket General Equilibrium

Consumer demand is modelled as budget-constrained utility maximization by a representative agent:

$$
\max u\left(x_{c}, y_{c}, E_{c}\right) \quad \text { s.t. } \quad p_{x} x_{c}+p_{y} y_{x}+\sum_{i} p_{i}^{E} E_{i}^{c}=M
$$

where $u$ denotes the utility from consumption of non-energy goods $x_{c}$ and $y_{c}$ as well as from the final energy consumption composite $E_{c} ; p_{x}, p_{y}$, and $p_{i}^{E}$ are the prices for non-energy and energy goods; $E_{i}^{C}$ refers to the final consumption demand of energy good $i$; and $M$ denotes the income of the representative household.

Consumer preferences which trade off composite final energy $E^{c}$ and non-energy goods at a constant elasticity of substitution (CES) are given as:

$$
u\left(x_{c}, y_{c}, E^{c}\right)=\left(\alpha E_{c}^{\left(1-\sigma^{C}\right)}+(1-\alpha)\left(x_{c}^{\theta^{C}} y_{c}^{\left(1-\theta^{C}\right)}\right)^{1-\sigma^{C}}\right)^{1 /\left(1-\sigma^{C}\right)}
$$

where $\sigma^{C}$ is the substitution elasticity; $\alpha$ denotes the distribtuion parameter; and $\theta^{C}$ is the value share of $x$ demand in the Cobb-Douglas $x y$-composite of final demand.

Substitution possibilities across energy goods in final demand are characterized by a CES function:

$$
E_{c}=\left(\sum_{i} \beta_{i} E_{i}^{c\left(1-\sigma^{E C}\right)}\right)^{1 /\left(1-\sigma^{E C}\right)}
$$

where $\beta_{i}$ is the distribution parameter; and $\sigma^{E C}$ denotes the elasticity of substitution.

Consumer income $(M)$ is determined by wages $w$, earnings $r$ on sector-specific capital 
and scarcity rents $u_{i t}$ on capacities of energy technology $t$ producing energy good $i$ :

$$
M=r_{x} \bar{K}_{x}+r_{y} \bar{K}_{y}+w \bar{L}+\sum_{i t} \mu_{i t} \bar{z}_{i t}
$$

where $\bar{K}_{y}, \bar{K}_{x}$ denote sector-specific (fixed) capital; $\bar{L}$ is the fixed labor supply; and $\bar{z}_{i t}$ denotes the capacity constraint on technology $t$ producing energy good $i$.

Goods $x$ and $y$ enter intermediate demand to energy production and final consumption demand:

$$
\begin{aligned}
& x=\sum_{i t} a_{i t}^{x} z_{i t}+x_{c} \\
& y=\sum_{i t} a_{i t}^{y} z_{i t}+y_{c}
\end{aligned}
$$

where $a_{i t}^{y}\left(a_{i t}^{x}\right)$ denote the (per-unit) input coefficient of non-energy input to the production of energy good $i$ by technology $t ; z_{i t}$ is the activity level of technology $t$ delivering energy good $i$.

Energy supplies enter as intermediate inputs into the production of non-energy goods and final demand. Furthermore energy supplies serve as intermediate inputs to the production of other energy goods. ${ }^{8}$ The market clearance condition for energy good $i$ is:

$$
E_{i}=E_{i}^{x}+E_{i}^{y}+E_{i}^{c}+\sum_{i^{\prime} t} b_{i i^{\prime} t} z_{i^{\prime} t}
$$

where $b_{i i^{\prime} t}$ is the input coefficient of energy good $i$ into technology $t$ producing energy good $i^{\prime}$.

The labor market is cleared by the real wage $w$ :

$$
L_{x}+L_{y}=\bar{L}
$$

Likewise, rental rates $r_{x}$ and $r_{y}$ clear sector-specific capital markets:

$$
K_{x}=\bar{K}_{x}
$$

$$
K_{y}=\bar{K}_{y}
$$

Upper bounds on energy sector technologies are realized through adjustment of technologyspecific rents $\mu_{i t}$ :

$$
0 \leq z_{i t} \leq \bar{z}_{i t}
$$

Production of non-energy goods $x$ and $y$ is based on profit maximization subject to technical constraints:

$$
\max p_{x} x-w L_{x}+\sum_{i} p_{i}^{E} E_{i}^{x} \quad \text { s.t. } \quad x=f_{x}\left(\bar{K}_{x}, L_{x}, g_{x}\left(E^{x}\right)\right)
$$

\footnotetext{
${ }^{8} \mathrm{Coal}$ and gas serve as inputs to the production of electricity, crude oil is refined into transportation fuels, etc.
} 
and

$$
\max p_{y} y-w L_{y}+\sum_{i} p_{i}^{E} E_{i}^{y} \quad \text { s.t. } \quad y=f_{y}\left(\bar{K}_{y}, L_{y}, g_{y}\left(E^{y}\right)\right)
$$

Three-level nested separable CES functions characterize trade-offs between primary factors and energy in the production of goods $x$ and $y$. At the top level, an energy composite is combined with a Cobb-Douglas aggregate in labor and capital subject to a constant elasticity of substitution:

$$
f_{i}\left(\bar{K}_{i}, L_{i}, E_{i}\right)=\phi_{i}\left(\gamma_{i} E_{i}^{\left(1-\sigma_{i}\right)}+\left(1-\gamma_{i}\right) \bar{K}_{i}^{\theta} L_{i}^{\left(1-\theta_{i}\right)\left(1-\sigma_{i}\right)}\right)^{1 /\left(1-\sigma_{i}\right)} i \in\{x, y\}
$$

where $\phi_{i}$ is the efficiency parameter, $\gamma_{i}$ is the distribution parameter, and $\sigma_{i}$ is the elasticity of substitution.

At the lower level, energy inputs are combined (to a sector-specfic energy input composite $\left.E_{i}\right)$ in a manner which distinguishes the differences in substitutability between electricitiy, coal, oil, and gas:

$$
E_{i}=E_{\mathrm{ELE}, i}^{\theta_{i}^{\mathrm{ELE}}}\left(\delta_{i} E_{\mathrm{COL}, i}^{\left(1-\sigma_{i}^{E}\right)}+\left(1-\delta_{i}\right)\left(E_{\mathrm{OL}, i}^{\theta_{i}, \text { oL }} E_{\mathrm{GAS}, i}^{\left(1-\theta_{i, \text { oL }}\right.}\right)^{\left(1-\sigma_{i}^{E}\right)}\right)^{\left(1-\theta_{i}^{\mathrm{ELE}}\right) /\left(1-\sigma_{i}^{E}\right)} i \in\{x, y\}
$$

where $\theta_{i}^{\mathrm{ELE}}$ is the value share of electricity in the Cobb-Douglas energy composite demand of sector $i ; \delta_{i}$ is the distribution parameter; $\theta_{i}^{\text {oIL }}$ refers to the value share of oil in the CobbDouglas oil-gas composite; $\sigma_{i}^{E}$ denotes the substituion elasticity between coal and the oil-gas composite.

Energy sector supplies are produced by profit-maximizing firms. The technology $t$ that produces energy good $i$ is then selected at a level which maximizes returns subject to capacity constraints:

$$
\max z_{i t}\left(p_{i}^{E}-p_{x} a_{i t}^{x}-p_{y} a_{i t}^{y}-\sum_{i^{\prime}} p_{i}^{E} b_{i^{\prime} i t}\right) \quad \text { s.t. } \quad z_{i t} \leq \bar{z}_{i t}
$$

\subsection{The Integrated Model as a MCP}

The model presented above omits a number of complications which arise in applied general equilibrium models. These might include multiple consumers with distinct preferences, taxes (on energy, goods or factor incomes), knowledge spillovers, or imperfect competition. In the absence of these features which typically violate integrability conditions, the integrated model can be solved as a conventional nonlinear program by maximizing $u$ subject to (6) through (16). The optimization approach is, however, often too restrictive in terms of the model features which need to be included for concrete policy analysis.

The complementarity format offers a flexible alternative to non-linear optimization as a means of representing economic equilibrium models through "canonical" general equillibrium conditions (see conditions (1), (2), and (3)). In the MCP framework, the algebraic representation of our stylised model begins from the dual cost minimization problems of the individual producers. For sectors $i=\{x, y\}$ we have cost-minimizing unit energy costs given by: 


$$
\begin{aligned}
p_{i}^{E}= & \left(\frac{p_{\mathrm{ELE}}}{\theta_{i}^{\mathrm{ELE}}}\right)^{\theta_{i}^{\mathrm{ELE}}}\left\{\delta_{i}\left(\frac{p_{\mathrm{COL}}}{\delta_{i}}\right)^{\left(1-\sigma_{i}^{E}\right)}\right. \\
& \left.+\left(1-\delta_{i}\right)\left[\left(\frac{p_{\mathrm{OIL}}}{\left(1-\delta_{i}\right) \theta_{i}^{\text {OIL }}}\right)^{\theta_{i}^{\text {oL }}}\left(\frac{p_{\mathrm{GAS}}}{\left(1-\delta_{i}\right)\left(1-\theta_{i}^{\text {OIL }}\right)}\right)^{\left(1-\theta_{i}^{\text {olL }}\right)}\right]^{\left(1-\sigma_{i}^{E}\right)}\right\}^{1 /\left(1-\sigma_{i}^{E}\right)}
\end{aligned}
$$

Unit profit functions for $x$ and $y$ are in turn given by:

$$
\begin{aligned}
\Pi_{i}= & p_{i}-\frac{1}{\phi_{i}}\left[\gamma_{i}\left(\frac{p_{i}^{E}}{\gamma_{i}}\right)^{\left(1-\sigma_{i}\right)}\right. \\
& \left.+\left(1-\gamma_{i}\right)\left(\frac{r_{i}}{\theta_{i}\left(1-\gamma_{i}\right)}\right)^{\theta_{i}\left(1-\sigma_{i}\right)}\left(\frac{w}{\left(1-\theta_{i}\right)\left(1-\gamma_{i}\right)}\right)^{\left(1-\theta_{i}\right)\left(1-\sigma_{i}\right)}\right]^{1 /\left(1-\sigma_{i}\right)}
\end{aligned}
$$

The unit cost of energy inputs to final demand are given by:

$$
p_{c}^{E}=\left(\sum_{i} \beta_{i}\left(\frac{p_{i}^{E}}{\beta_{i}}\right)^{1-\sigma^{E C}}\right)^{1 /\left(1-\sigma^{E C}\right)}
$$

and the resulting cost of a unit of final consumption is:

$p^{c}=\left[\alpha\left(\frac{p_{c}^{E}}{\alpha}\right)^{1-\sigma^{C}}+(1-\alpha)\left(\left(\frac{p_{x}}{\theta^{C}(1-\alpha)}\right)^{\theta^{C}}\left(\frac{p_{y}}{\left(1-\theta^{C}\right)(1-\alpha)}\right)^{\left(1-\theta^{C}\right)}\right)^{1-\sigma^{C}}\right]^{1 /\left(1-\sigma^{C}\right)}$

Finally, the unit profit associated with technology $t$ for energy good $i=\{c o l$, oil, gas, ele $\}$ is:

$$
\Pi_{i t}^{E}=p_{i}^{E}-p_{x} a_{i t}^{x}-p_{y} a_{i t}^{y}-\sum_{i^{\prime}} p_{i}^{E} b_{i^{\prime} i t}-\mu_{i t}
$$

Given the underlying functional forms, we observe that the complementarity conditions only will apply for the energy sector technologies and the shadow prices on the associated capacity constraints; all of the macro economic prices and quantities will be non-zero. By use of Shepard's Lemma we can then write the equilibrium as the following mixed complementarity problem:

- Zero-profit conditions:

$$
\begin{gathered}
\bar{z}_{i t} \geq z_{i t} \perp \mu_{i t} \geq 0 \\
-\Pi_{i t}^{E} \geq 0 \perp z_{i t} \geq 0 \\
\Pi_{x}=0 \\
\Pi_{y}=0
\end{gathered}
$$


- Market clearence conditions:

$$
\begin{aligned}
& x=\sum_{i t} a_{i t}^{x} z_{i t}+c \frac{\partial \Pi_{c}}{\partial p_{x}} \\
& y=\sum_{i t} a_{i t}^{y} z_{i t}+c \frac{\partial \Pi_{c}}{\partial p_{y}} \\
& \bar{L}=x \frac{\partial \Pi_{x}}{\partial w}+y \frac{\partial \Pi_{y}}{\partial w} \\
& \bar{K}_{x}=x \frac{\partial \Pi_{x}}{\partial r_{x}} \\
& \bar{K}_{y}=y \frac{\partial \Pi_{y}}{\partial r_{y}} \\
& \sum_{t} z_{i t}-\sum_{i^{\prime} t} b_{i i^{\prime} t} z_{i^{\prime} t}=c \frac{\partial \Pi_{c}}{\partial p_{i}^{E}}+x \frac{\partial \Pi_{x}}{\partial p_{i}^{E}}+y \frac{\partial \Pi_{y}}{\partial p_{i}^{E}} \\
& c=\frac{M}{p_{c}}
\end{aligned}
$$

- Income balance:

$$
M=r_{x} \bar{K}_{x}+r_{y} \bar{K}_{y}+w \bar{L}+\sum_{i t} \mu_{i t} \bar{z}_{i t}
$$

Table 1 provides a summary of the variables appearing in the integrated model.

Table 1: Equilibrium Variables in the Integrated Model

\begin{tabular}{|cl|}
\hline \multicolumn{1}{|c|}{ Activity variables } \\
\hline \hline$c$ & Aggregate consumption \\
$x, y$ & Production of goods $x$ and $y$ \\
$E_{i}$ & Aggregate output of energy good $i$ \\
$z_{i t}$ & Production by technology $t$ for energy good $i$ \\
$E_{i}^{x}, E_{i}^{y}$ & Demand for energy good $i$ in sectors $x$ and $y$ \\
$E_{i}^{c}$ & Final demand for energy good $i$ \\
$L_{x}, L_{y}$ & Labor demand in goods $x$ and $y$ \\
\hline \hline & $\quad$ Price variables \\
\hline \hline$p_{c}$ & Price index of final consumption \\
$p_{x}, p_{y}$ & Non-energy goods $x$ and $y$ \\
$p_{i}^{E}$ & Energy prices for $i=\{$ OIL, GAS, COL, ELE $\}$ \\
$w$ & Wage rate \\
$r_{x}, r_{y}$ & Returns to non-energy capital \\
$\mu_{i t}$ & Energy sector rents \\
\hline \hline \multicolumn{2}{c}{ Income variable } \\
\hline \hline$M$ & Income of representative agent \\
\hline
\end{tabular}




\subsection{Decomposition}

Our decomposition strategy requires that we separate the integrated model into a top-down model of the overall economy and a bottom-up model of the energy supply system. Within the top-down model, we treat net energy system netputs as exogenous. Energy supply activities are no longer endogenous and we can drop equations (17) and (18). Net energy supplies and inputs of non-energy goods to the energy system enter the top-down model as parameters. Parameterized energy-sector netputs $\tilde{S}_{i}$ and inputs $\tilde{x}_{E}$ and $\tilde{y}_{E}$ are valued at market prices which implicitly include rents on specific energy resources (so we can drop these from the income constraint). The adjusted market clearance condition for energy goods within the top-down model is:

$$
\tilde{S}_{i}=E_{i}^{x}+E_{i}^{y}+E_{i}^{c}
$$

and the revised market clearance conditions for non-energy goods are:

$$
x=\tilde{x}_{E}+c \frac{\partial \Pi_{c}}{\partial p_{x}}
$$

and

$$
y=\tilde{y}_{E}+c \frac{\partial \Pi_{c}}{\partial p_{y}}
$$

The revised income balance (28) reads:

$$
M=r_{x} \bar{K}_{x}+r_{y} \bar{K}_{y}+w \bar{L}+\sum_{i} p_{i}^{E} \tilde{S}_{i}-p_{x} \tilde{x}_{E}-p_{y} \tilde{y}_{E}
$$

The bottom-up model can be represented as a quadratic programming problem in which the sum of producer and consumer surplus is maximized subject to supply-demand balances for energy and resource bounds on technologies:

$$
\max \sum_{i} \tilde{p}_{i}^{E}\left(1+\frac{2 \tilde{S}_{i}-S_{i}}{2 \epsilon_{i} \tilde{S}_{i}}\right)-\tilde{p}_{x} x_{E}-\tilde{p}_{y} y_{E}
$$

subject to

$$
\begin{gathered}
S_{i}=\sum_{t} z_{i t}-\sum_{i^{\prime} t} b_{i i^{\prime} t} z_{i^{\prime} t} \\
x_{E}=\sum_{i t} a_{i t}^{x} z_{i t} \\
y_{E}=\sum_{i t} a_{i t}^{y} z_{i t} \\
0 \leq z_{i t} \leq \bar{z}_{i t}
\end{gathered}
$$

Table 2 summarizes the additional variables and parameters appearing in the bottom-up (sub-)model. 
Table 2: Variables and Parameters in the Decomposed Model

\begin{tabular}{|cl|}
\hline \multicolumn{1}{|c|}{ Variables } \\
\hline \hline$S_{i}$ & Net supply of energy good $i$ \\
$x_{E}, y_{E}$ & Aggregate demand for $x$ and $y$ as inputs to energy production \\
$z_{i, t}$ & Activity level of technology $t$ producing energy good $i$ \\
\hline \hline & \multicolumn{1}{c|}{ Parameters } \\
\hline \hline$\tilde{S}_{i}$ & Reference level of demand or supply for energy good $i$ \\
$\tilde{p}_{i}^{E}$ & Reference price of energy good $i$ \\
$\tilde{p}_{x}, \tilde{p}_{y}$ & Reference prices of non-energy goods $x$ and $y$ \\
$\epsilon_{i}$ & Demand elasticity for energy good $i$ \\
\hline
\end{tabular}

\subsection{Parameterization}

Before we perform numerical simulations we parameterize our stylized model with economic data. The benchmark statistics are given in Table 3 in terms of a social accounting matrix King [1985]. In the initial aggregate dataset energy constitutes $10 \%$ of overall final demand. ${ }^{9}$

The bottom part of Table 3 provides details on the energy demand structure in sectors $x$ and $y$ as well as in final demand. The column labelled " $e$ " summarizes total energy supplies by energy carrier $i$ and non-energy inputs to energy production (in our case there are only inputs of the macro good $y$ ).

Table 3: Base Year Social Accounts

\begin{tabular}{|l|cccc|}
\hline & $x$ & $y$ & $e$ & $f d$ \\
\hline$x$ & 15 & & & -15 \\
$y$ & & 100 & -24 & -76 \\
$l$ & -5 & -40 & & 45 \\
$k$ & -5 & -50 & & 55 \\
\hline ele & -2 & -5 & 10 & -3 \\
oil & -1 & -2 & 7 & -4 \\
gas & -1 & -1 & 3 & -1 \\
col & -1 & -2 & 4 & -1 \\
\hline
\end{tabular}

\begin{tabular}{ll} 
Key & \\
\hline$x:$ & energy intensive production \\
$y:$ & macro production \\
$e:$ & energy production \\
$f d:$ & final demand \\
ele: & electricity \\
oil: & oil \\
gas: & gas \\
col: & coal
\end{tabular}

We have implemented a generic procedure how the aggregate description of the economy's energy supply side can be further detailed through a discrete representaiton of energy supply technologies thereby warranting consistency with the aggregate data set as given in Table 3. First, one can specify the desired number of technologies which are available for the generation of energy commodities. ${ }^{10}$ In a second step, the program randomly generates a

\footnotetext{
${ }^{9}$ We have parameterized the energy intensity of final demand within our computer programs to investigate how the convergence of the decomposition procedure depends on the size of the energy sector - see Figure 6 .

${ }^{10}$ We can thus perform sensitivity analysis on the computational gains/cost of the decomposition method with respect to the number of technologies.
} 
cost distribution for each technology thereby assigning a certain fraction of technologies as initially idle (non-profitable) at benchmark prices. The cost structure of discrete technologies - fuel costs and non-energy input costs - is then again assigned randomly; capital earnings, i.e. scarcity rents on technological capacities, are determined as a residual (if a technology is initially idle, the initial rents are obviously zero). Finally, relative capacities are randomly assigned and scaled such that net energy supply equals the given overall economic energy demand (see Table 3).

Figure 4 illustrates the step-wise energy supply structure by technologies in merit order for the different energy commodities (the number of available technologies has been set to 10 in this example). At benchmark energy prices of unity, the break-even "point" for technologies is at marginal costs of 1 - all technologies that are more expensive will be initially inactive.

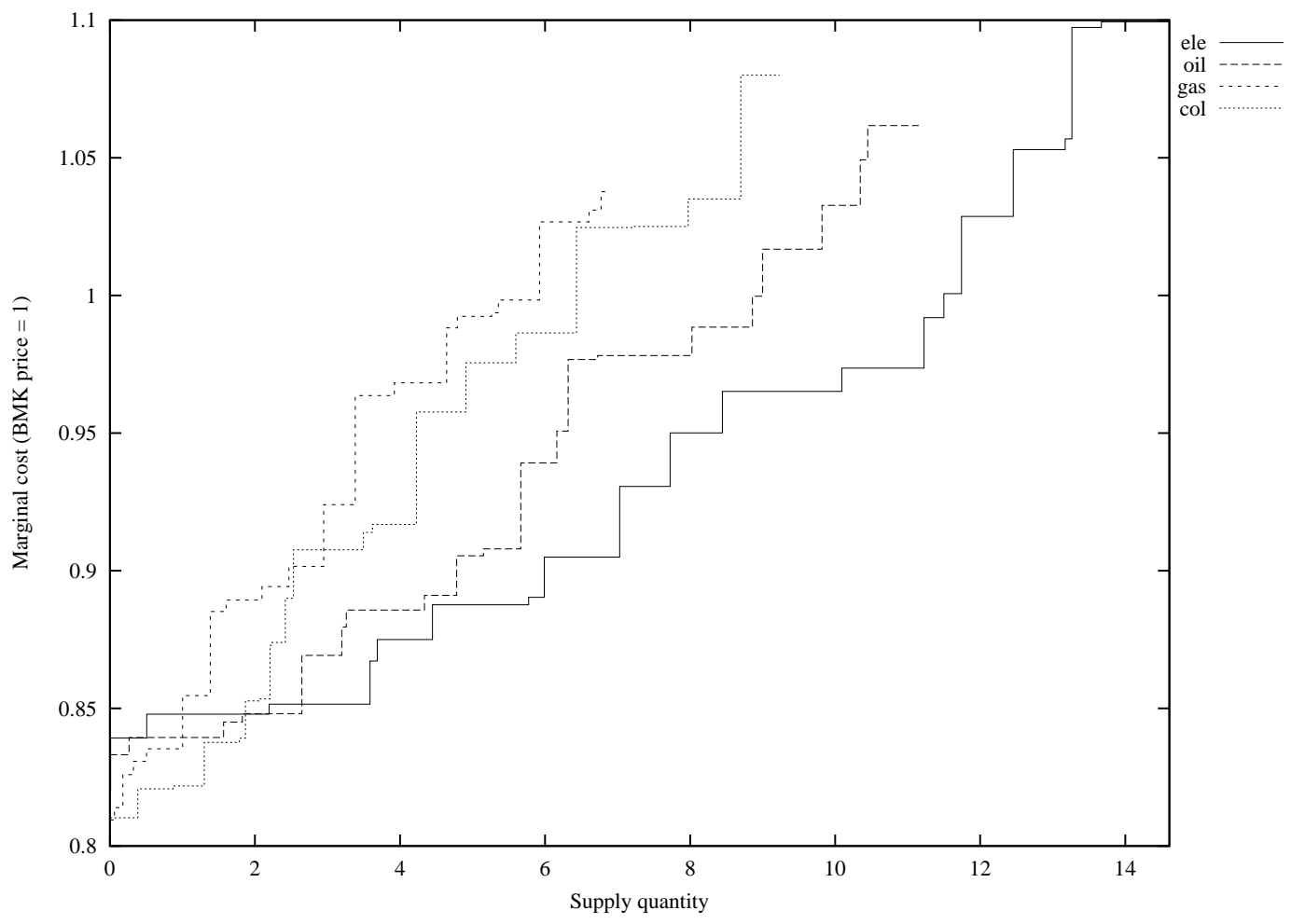

Figure 4: Energy Supply Schedules (Number of Technologies $=20$ )

\subsection{Computational Results}

In order to illustrate the potential usefulness of our decomposition approach, we provide some numerical tests with the calibrated version of our stylized model. As an exogenous policy shock, we assume a technological breakthrough which reduces non-energy inputs to non-fossil electricity generation technolgies by a half. 
The decomposed integrated model is solved iteratively in its top-down and bottom-up sub-models. After the exogenous policy shock, we may first solve the top-down model. Next, we solve the bottom-up model taking into account the equilibrium prices of the top-down model. The solution values of the bottom-up model are subsequently used to update the quantities on energy system outputs and inputs which enter into the top-down model.

Figure 5 provides some evidence on the computational efficiency of our decomposition algorithm. We compare the computational cost for the integrated model formulation vis-vis the decomposed model formulation as a function of the number of energy technologies. It becomes evident that the decomposition delivers substantial time savings towards an increasing number of technologies.

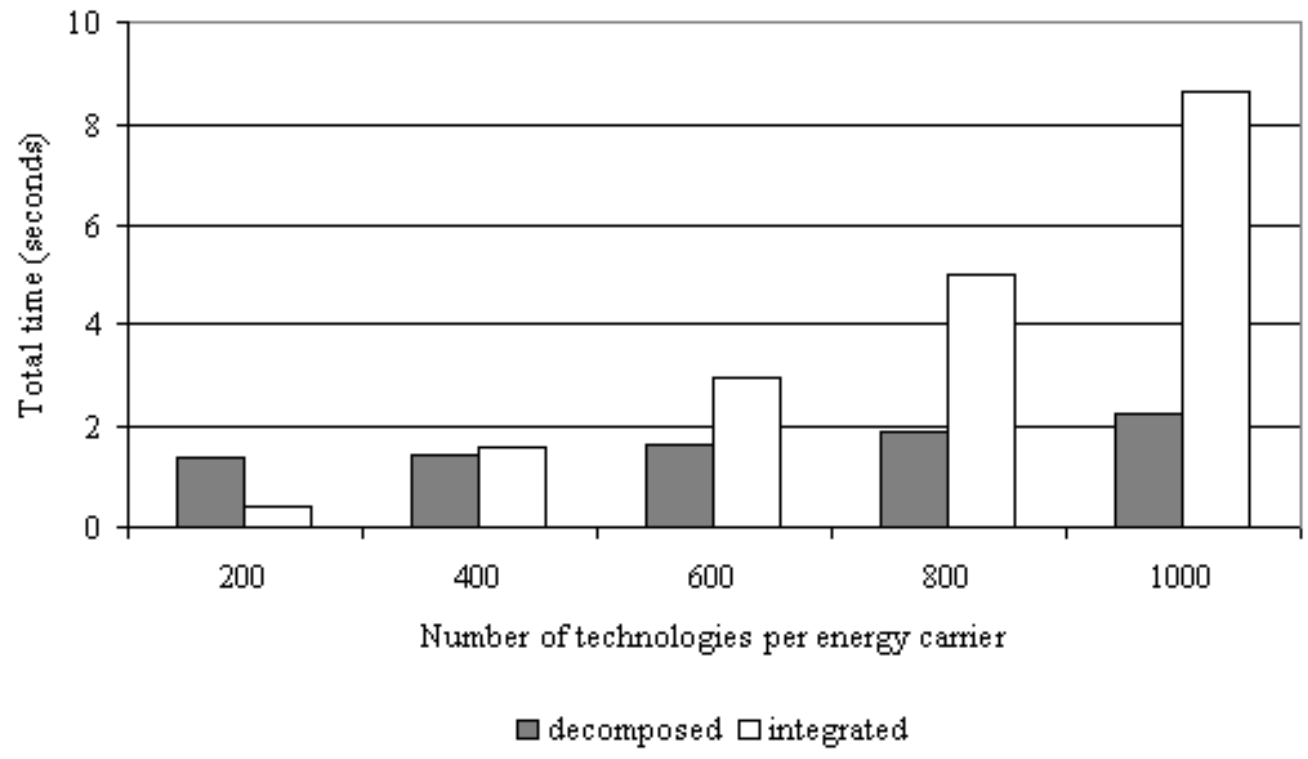

Figure 5: Computational Cost of Decomposition

Figure 6 investigates the convergence performance of the decomposed model with respect to the energy intensity of final demand. The graph shows how iterations required for convergence increase with the size of the energy sector.

\section{Conclusions}

The combination of bottom-up and top-down approaches constitutes a long-standing challenge in applied energy policy analysis. The formulation of economic equilibrium conditions as mixed complementarity problem provides a unifying framework for combining technological details of bottom-up models and economic richness of top-down models. However, 


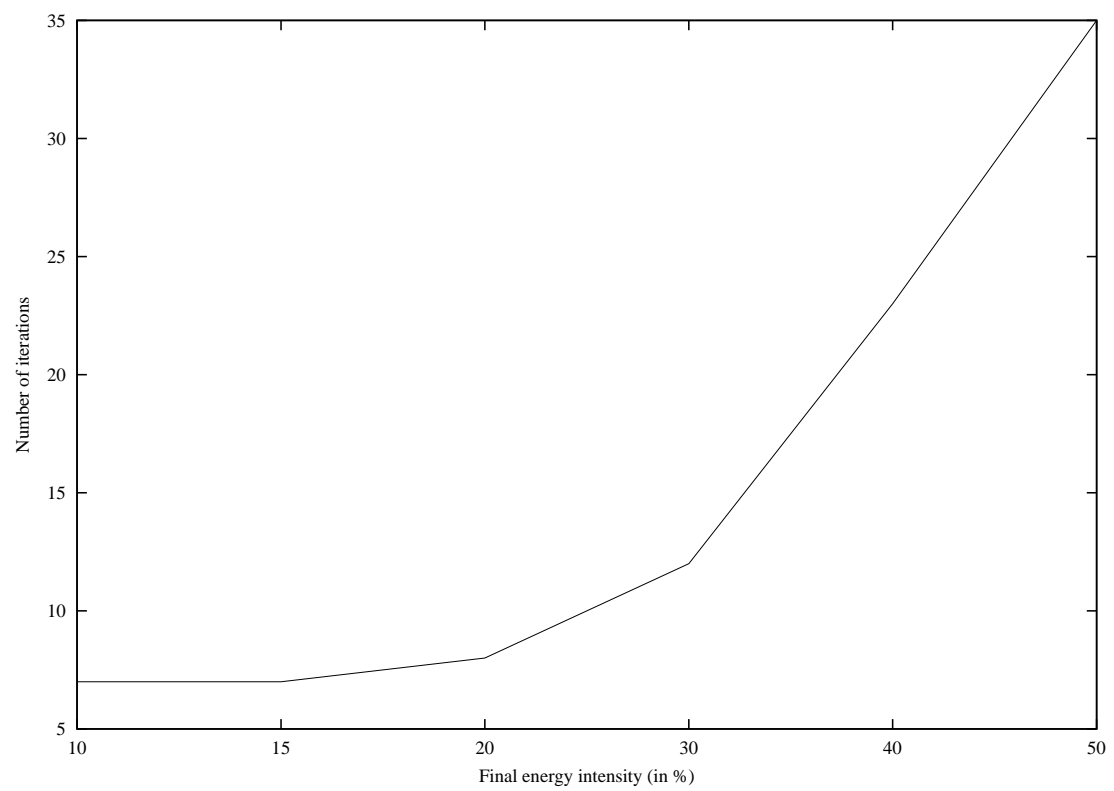

Figure 6: Convergence Performance of Decomposed Model as a Function of Energy Intensity

dimensionality may impose significant limitations on the practical application of the integrated MCP framework, particularly when the underlying programming formulation of the energy system includes both upper and lower bounds on many decision variables.

In this paper, we have presented a decomposition approach that combines different mathematical formats - mixed complementarity and mathematical programming - thereby taking advantage of efficient and robust solution algorithms for each format: We use complementarity methods to solve the top-down economic equilibrium model and quadratic programming to solve the underlying bottom-up energy supply model.

Our decomposition technique overcomes dimensionality restrictions of the integrated MCP framework and can be readily applied to the combination of large-scale top-down and bottom-up models for comprehensive energy policy analysis. 


\section{References}

Bergmann, L., "The Development of Computable General Equilibrium Models," in L. Bergman, D. W. Jorgenson, and E. Zalai, eds., General Equilibrium Modeling and Economic Policy Analysis, Cambridge, 1990, pp. 3-30.

Böhringer, C., "The Synthesis of Bottom-Up and Top-Down in Energy Policy Modeling," Energy Economics, 1998, 20 (3), 233-248.

_ _ A. Müller, and M. Wickart, "Economic Impacts of a Premature Nuclear Phase-Out in Switzerland," Swiss Journal of Economics and Statistics, 2003, 139 (4), 461-505.

and T.F. Rutherford, "Integrating Bottom-Up into Top-Down:A Mixed Complementarity Approach," Discussion Paper No. 05-28, ZEW, Mannheim 2005.

Cottle, R. W. and J.-S. Pang, The Linear Complementarity Problem, Academic Press, 1992.

Dirkse, S. and M. Ferris, "The PATH Solver: A Non-monotone Stabilization Scheme for Mixed Complementarity Problems," Optimization Methods $\mathcal{E}$ Software, 1995, 5, $123-156$.

Frei, C.W., P.-A. Hadi, and G. Sarlos, "Dynamic formulation of a top-down and bottom-up merging energy policy model," Energy Policy, 2003, 31 (10), 1017-1031.

Hudson, E.A. and D.W. Jorgenson, "US energy policy and economic growth, 19752000," The Bell Journal of Economics and Management Science, 1974, 5, 461-514.

International Panel on Climate Change (IPCC), Climate Change, 1995: Economic and Social Dimensions of Climate Change - Contribution of Working Group II to the Second Assessment Report of the Intergovernmental Panel on Climate Change, Cambridge, 1996.

King, B., "What is a SAM?," in "Social Accounting Matrices: A Basis for Planning," Washington D. C.: The World Bank, 1985.

Manne, A.S. and T.F. Rutherford, "International trade, capital flows and sectoral analysis: formulation and solution of intertemporal equilibrium models," in W.W. Cooper and A.B. Whinston, eds., New Directions in Computational Economics, Springer, 1994, pp. 191-205.

Mathiesen, L., "Computation of Economic Equilibrium by a Sequence of Linear Complementarity Problems," in A. Manne, ed., Economic Equilibrium - Model Formulation and Solution, Vol. 23 1985, pp. 144-162.

Rutherford, T. F., "Extensions of GAMS for Complementarity Problems Arising in Applied Economics," Journal of Economic Dynamics and Control, 1995, 19, 1299-1324. 